$\begin{gathered}\text { EPiC Series in Built Environment } \\ \text { Volume 2, 2021, Pages 18-27 }\end{gathered}$
ASC 2021. 57th Annual Associated Schools
of Construction International Conference

\title{
The Dark Side of the Drones: A Review of Emerging Safety Implications in Construction
}

\author{
Mohammad Khalid, B.Sc., Mostafa Namian, Ph.D., Carol Massarra, Ph.D. \\ East Carolina University \\ Greenville, North Carolina
}

The construction industry has experienced a significantly rapid integration of Unmanned Aerial Vehicles (UAVs), (known as drones) within multifarious construction applications due to their prevalent stature in accessibility, reliability, accuracy, and low-cost. Given the dynamic nature of the hazard-prone construction sites, the rapid proliferation of UAVs potentially incorporates additional operational complexities, hazards, and associated safety risks. These safety implications are emerging in the absence of construction-specific UAV-standard guidelines and strategies for effective risk mitigation. Despite the remarkable benefits, UAVs can expose workers to a wide range of unwarranted workplace hazards, especially when operating around densely populated construction sites. This study aims to (1) explore the current understanding of the UAV-associated safety risks and (2) identify the potential risks to which construction workers are exposed. Collisions with workers and properties have been found to be the key safety risks of UAV adoption. In this context, due to the scarcity of studies, there is a substantial lack of construction-based safety understanding relevant to UAV applications. It is expected that the industry professionals and practitioners can benefit from the presented information to effectively address the safety risks and develop appropriate control measures to ensure safe operation by preventing accidents.

Key words: Unmanned Aerial Vehicle; UAV; Safety Risk; Hazard; Construction Safety.

\section{Introduction}

Construction can clearly be indicated as one of the most hazardous industries having the greatest number of occupational fatal accidents (1,061 fatalities recorded in 2019) every year (U.S. Bureau of Labor Statistics, 2020). The complexity of construction jobs is largely responsible for the wide spectrum of safety hazards (Trinh \& Feng, 2020). The construction industry involves a complex and dynamic working environment and despite the considerable extent of safety-related improvements in the last decade (Hinze, Thurman, \& Wehle, 2013), occupational accidents are repeatedly occurring (Guo, Yiu, \& González, 2015). The recent technological advancement in the form of Unmanned Aerial Vehicles (UAVs) has aided in alleviating the complex and hazardous working conditions. Accordingly, UAVs are in a phase of expeditious proliferation because this version of aircraft does not require an on-board pilot which results in critical and dangerous tasks for the construction workplace becoming safer than 
before (Gheisari \& Esmaeili, 2019). However, this rapid growth of UAVs brings in unwarranted workplace hazards to the workers and properties by exposing them to a wide range of safety risks on construction sites.

Due to advantages such as accessibility, reliability, accuracy, and low-cost, UAVs are being heavily integrated in construction applications to improve efficiency, economy, and safety of the jobsite. The ability to control the aircraft remotely backed up by improved accuracy has attracted people from diverse backgrounds. Nevertheless, the popularity of UAVs does not necessarily supersede the emerging safety threats. The primary safety risk of UAVs operated in construction sites is collision with workers and properties. Ranging from a minor bruise to a catastrophic fire hazard, the UAV retains the potential to risk all of them (Namian, Khalid, Wang, \& Turkan, 2021a). The negative impacts of adopting UAVs on the jobsite remain unnoticed due to the scarcity of research to effectively identify and address the UAV associated safety risks. Unidentified safety hazard is a prime source of fatal accidents and the hazards typically remain unrecognized by the majority of the construction workers. Therefore the severity of the risks become even greater because of the failure to realize them before they actually happen (Namian, Zuluaga, \& Albert, 2016).

Research on UAVs in construction applications have focused on UAVs beneficial outcomes and opportunities rather than on the negative safety impacts. Therefore, UAVs integration in construction has not effectively been a subject of scrutiny that can lead to the development of comprehensive understanding and safety management strategies for mitigating the safety challenges. However, it is important to understand that UAV technology is still new, and the entirety of the safety threats are yet to be explored and managed. Specifically, this review paper aims to (1) explore the current understanding of the UAV-associated safety risks and (2) identify the potential risks to which construction workers are exposed.

\section{Research Methodology}

This research study performed a holistic review of existing literature by applying a systematic search approach and extensively identifying relevant publications from the Google Scholar database. The aims of the study were set as targets and they were disintegrated into individual concepts and related keywords. The following search syntaxes were searched for the study retrieval: "Unmanned Aerial System" or "Unmanned Aerial Vehicles" or "Drone" or "UAV" or "UAS" or "Safety" or "Hazard" "Risk" or "Construction". A total of 31 research articles were collected to review. A review of the references from those articles led towards manual identification of 4 government websites for specific information retrieval.

\section{Construction Applications of UAVs}

The offerings of UAVs take pride in making applications safer and easier in cases where the tasks may seem dangerous and critical for human beings. To understand the inclusiveness of this technology integration in construction, this review takes a step forward in exploring the versatile applications along with the never-seen-before safety risks introduced by the UAVs. The UAVs can carry out remote surveillance by more easily accessing hazardous locations, tracking resources, and inspecting safety issues eliminating the need for employees to operate in unsafe conditions (Kas \& Johnson, 2020). Remote sensing and photogrammetry software features are enabling UAVs to collect data through realtime transmission and processing for construction building information and surveying applications (Wang et al., 2016). Moreover, UAV-assisted applications such as facility management, damage 
evaluation, progress monitoring, and 3D modeling have already become common across many construction job sites.

\section{FAA's Stance in Accountability and Regulations}

The Federal Aviation Administration (FAA) reported that 1.5 million UAVs and 160,000 remote pilots are currently registered in their system which indicates the rapid growth of UAV adoption and heavy usage over the last decade (Federal Aviation Administration, 2019). In accordance with the present condition, FAA Aerospace Forecast suggests an upsurge in UAV usage in the upcoming fiscal years of 2020 to 2040 due to the recreational and commercial applications facilitated by the improved production technology and falling equipment prices (FAA Aerospace Forecasts, 2020).

The FAA is responsible for introducing and implementing strategies to regulate the usage of UAVs in airspaces to ensure the safe well-being and security of the general public. To control the usage of UAVs, the FAA has necessitated the operators obtain the pilot license and the UAVs weighted between 0.55 to 55 pounds to be registered. Under the FAA's small UAS rule (Part 107), the flight speed is limited to $100 \mathrm{mph}$ and the flying altitude at 400 feet. FAA has recently introduced the remote identification (remote ID) for UAVs to retrieve information on UAVs identification, location, altitude, and control station. This system essentially helps the FAA, federal, and law enforcement agencies to identify unsafe UAV operations and take appropriate actions (Federal Aviation Administration, 2019). According to section 14 C.F.R. Part 107, FAA implements restrictions in flying UAV over people (Occupational Safety and Health Administration, 2018) which bears a possible resemblance to the densely populated construction sites.

\section{Overview of Operational Complexities}

Despite the UAVs' beneficial contribution, there is a myriad of operational difficulties related to this new technology, and very few preventive countermeasures are taken into consideration for effective risk mitigation. As the pool of UAV applications becomes broader trying to meet the expanded commercial demands, the risk of operational complexities is leaning towards a more serious and consequential outcome. Therefore, the underlying safety risks associated with UAVs' operational limitations are becoming significantly dangerous for working personnel as the usage of UAVs in construction is growing every day. The UAVs were primarily introduced to the construction jobs for surveillance and monitoring purposes. Nowadays, the applications have achieved a multi-faceted progression for excellent prospects supported by continuous developments.

UAVs may experience a diversified combination of operational constraints ranging from their systemrelated malfunctions to endurance against external setbacks that are directly or indirectly responsible for reduced accuracy and reliability (Schenkelberg, 2016). The Aircraft's loss of control (LOC) is a leading cause for system-related failures resulting in accidents and crashes which can be generated from signal interference or human error (Parush, 2006). Factors such as limited operating flight envelope, onboard system error, flight crew shortcomings, vehicular impairment conditions, adverse weather or extreme atmospheric interference, and restricted visibility primarily contribute to the list of LOC-related aircraft accidents (Belcastro et al., 2014). The deficiency of a fool-proof UAV onboard system capable to withstand dynamic operational challenges can potentially lead to unexpected actions such as flight instability, unintentional landing, and inability to cope with diverse environmental conditions (York, Al-Bayati, \& Al-Shabbani, 2020). UAVs are susceptible to signal interference or 'GPS-spoofing' that 
can lead to lost control. In the worst case scenario, it can let hijackers take control of the operational aircraft to deviate from the original flight-plan and cause nuisance (Rao, Gopi, \& Maione, 2016).

The integration of newer UAV models and corresponding architecture variances are responsible for the human-machine interactive failures leading to more human errors and associated accidental outcomes (Neff \& Garman, 2020). The low flight dependability of UAVs limits their operability, especially they can be extremely vulnerable to adverse weather conditions such as strong air streams, potentially escalating the precariousness for the UAV pilots (Charlesraj \& Rakshith, 2020). Typical UAVs lack the provision to be equipped with long-haul battery life due to limited onboard allocation intended for the weight reduction purpose. They usually consist of a battery life that lasts less than half an hour (Menouar et al., 2017). High consumption of onboard power with limited battery resources is a challenge for UAV pilots; this leads to shorter flight time and accidental flight abruption.

\section{Understanding the Potential Safety Risks}

There has been significant research concerning the technological advancement and beneficial attributes of UAVs. However, advanced research concerning the inherent safety hazards and associated risks of UAVs is still scarce. The concealed safety risks can be pro-actively minimized if detected at the appropriate stage before mass integration into day-to-day construction applications. A UAV consists of elements that can have adverse impacts and collateral damages on people and properties during its flight (Belcastro et al., 2017). The UAVs typically consist of minimal safety features making them vulnerable to system-related mechanical malfunctions such as rapid power discharge or premature crash-landing during operations. With the increase in production, the manufacturing costs have been marked down significantly occurring a shift up in the functionality-to-cost ratio which has further limited the provisions of safety features (Clarke, 2014). For example, the high-speed spinning blades can come in contact with humans or the UAV itself can fall from the sky with hardly any warning, containing high possibilities of injuries such as blunt head trauma or bruises (Afman et al., 2018). Besides humans, an operational UAV subjected to system malfunction or piloting error can impose a high risk of collision with the ground properties or critical structures in the construction job sites heightening the chance of major financial loss and productivity halt (McCabe, Hamledari, Shahi, Zangeneh, \& Azar, 2017). These sorts of accidental outcomes may occur because of losing control of the UAV and setting it off-course from the pre-planned flight path.

Construction projects in a controlled environment such as confined spaces or oil-petrochemical plants bear a significant risk from UAV assisted applications. A source of ignition (e.g., spark caused by rotating blades of UAVs involved in a collision with any metal surface) can encounter existing combustible dust particles resulting in dangerous dust explosion accidents (Abuswer, Amyotte, Khan, \& Morrison, 2013). Besides, onboard lithium battery cartridges and liquid fuel resources for powering the propulsion of UAVs are practicable sources of potential fire hazard (Opfer \& Shields, 2014). The following Table 1 depicts a summary of the UAV-associated operational and safety challenges that can materialize in construction sites during flight operations. These issues may endanger workers and properties in construction environments if not managed at the appropriate stages.

Table 1

Summary of UAV-associated operational and safety challenges

Type of Challenge Description 


\begin{tabular}{ll}
\hline UAV Selection & $\begin{array}{l}\text { Selecting appropriate UAV models equipped with proper gears (non-spark } \\
\text { materials or protective bumper to reduce collision impact) }\end{array}$ \\
\hline Limited Power & $\begin{array}{l}\text { Limited battery resource causing unexpected termination of flight leading } \\
\text { to crashes }\end{array}$ \\
\hline Element Degradation & Degradation of battery, rotors, screws, and sensors over time \\
\hline On-board Power & Built-in lithium battery subjected to overheat leading to fire hazard \\
\hline Signal Interference & $\begin{array}{l}\text { Usage of communication devices (2-way radio communicators) and GPS- } \\
\text { spoofing interfering with UAV operating signals }\end{array}$ \\
\hline Loss of Control & $\begin{array}{l}\text { Losing control over the UAV leading to crash and collision with people or } \\
\text { properties }\end{array}$ \\
\hline Behavioral Limits & $\begin{array}{l}\text { Erroneous actions performed by flight crew due to intense workload, } \\
\text { distraction, or fatigue }\end{array}$ \\
\hline External Interference & $\begin{array}{l}\text { Curious workers, birds, vehicles, and aircrafts interfering with ongoing } \\
\text { UAV operation }\end{array}$ \\
\hline Environmental & $\begin{array}{l}\text { Loss of control due to adverse weather (heavy wind, intense rainfall, fog, } \\
\text { and snow) }\end{array}$ \\
\hline Conditions & Disruption of workers' attention from their monotonous jobs \\
\hline Distraction & Workers can generate psychological stress from UAVs flying near them \\
\hline Inadequate Training & $\begin{array}{l}\text { Emergent confusion in workers regarding role of duty due to insufficient } \\
\text { training and hand-on familiarity of UAVs }\end{array}$ \\
\hline Piloting Error & Flight crew's lack of experience and intuitive decision-making skills \\
\hline Privacy & $\begin{array}{l}\text { Collection of sensitive information risking privacy violation (workers' } \\
\text { location and activities, surrounding properties' footage) }\end{array}$ \\
\hline Trespassing & $\begin{array}{l}\text { Flying over private properties around the target proximity of construction } \\
\text { sites or external rogue UAVs flying into construction sites }\end{array}$ \\
\hline
\end{tabular}

\section{Distraction by the UAVs}

UAVs function as a bridge of socio-technical network, which also serves as the relational part of humanmachine interaction with the potential of having a significant influence on any community (Benjamin, 2020). A viable form of safety risk is the workplace distraction by the UAVs which can possibly result in severe accidents by impairing the basic cognitive functions of working personnel. Even UAV pilots can be subject to cognitive overload that can reduce their ability to perform safely leading to erroneous piloting commands in operation and endangering human lives and properties (Coker, 2013). A construction job site is, by its nature, a hazard-prone workplace that is already filled with numerous safety hazards and workers must use their essential cognitive capabilities involving the combination of perception, memory, thought-processing and, decision-making aptitudes (Zhang, Murphy, Fang, \& Caban-Martinez, 2015). Research also suggests that distraction reserves the ability to undermine basic cognitive functions such as attention and situational awareness that are fundamental for a construction worker to perceive risks and recognize hazards in the workplace (Craik, 2014; Namian, Albert, \& Feng, 2018). Figure 1 shows an example of distracted workers. 


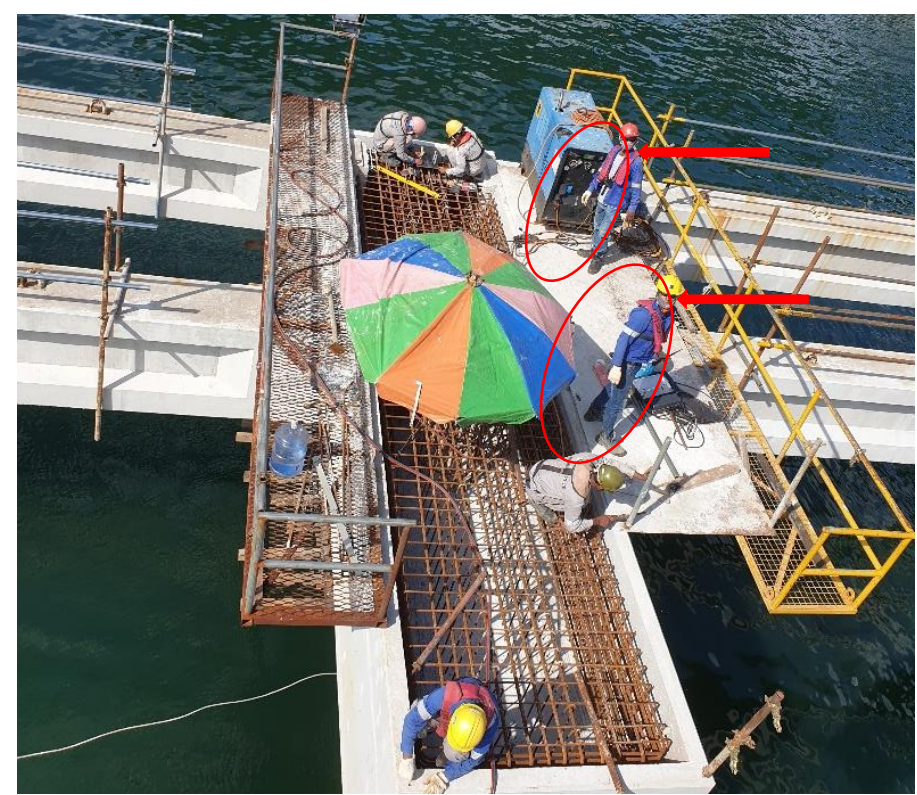

Figure 1. An example of distracted workers during an ongoing construction task (Image by Mohammad Khalid)

UAVs are capable to disrupt and seize the attention of the construction personnel during their taskspecific job performance (Martinez, Gheisari, \& Alarcón, 2020). Impeding concentration from a construction activity requiring maximum physiological and cognitive function can lead a worker to perceptual misinterpretation of risks which can potentially contribute to fatal consequences.

\section{Privacy and Ethical Implications of UAVs}

As UAVs are gaining widespread approval within construction-related operations, the question of the legal framework and privacy demands validated answers to protect the stakeholders from unforeseen risks. There are potential consequences pertaining to privacy invasion while UAVs are made airborne to serve commercial or entertainment purposes. Moreover, UAVs operation can bring in ethical and privacy implications concerning exploitation of sensitive information such as video footage or images of workers, in cases where strict professional confidentiality is violated. UAVs can also be subject to unauthorized trespassing while flown over the construction site or its proximity for collecting information. The psychological stress generated from UAVs trespass or privacy violation can overwhelm the construction workers which may result in erroneous and unsafe decisions on the job site.

\section{Current State of UAV-based Safety Perception}

Despite the predominant integration of UAVs in construction applications, it is evident that the majority of construction personnel, specifically workers are aware but still not adequately familiarized with the diversified characteristics of UAVs (Charlesraj \& Rakshith, 2020). (Namian, Khalid, Wang, \& Turkan, 2021b) has analyzed a trend of UAV-related risk perception based on construction personnel's years of professional experience. They suggest that individuals with up to 2 years of construction experience 
perceive a high level of risk and individuals having more than 30 years of experience similarly exhibit a high-risk perception as well. This research has also identified a statistically significant difference between the safety risk perception based on the factor of having an FAA issued UAV operating license. Moreover, this difference can be linked to the 'overconfidence bias' phenomena that is present among the FAA licensed pilot who overestimates the safety risks of UAVs due to their prior knowledge and experience.

\section{Developed Measures to Mitigate UAV Risks}

A growing number of research studies have attempted to develop methods to minimize the risks introduced by UAVs in civilian applications. Blimps and airbag systems have been installed on UAVs targeting to reduce the impact of collisions with people or properties in closed or populated environments (Asadi et al., 2020). Network or signal transmission interference is a glaring problem in UAV operations (Kovacs, Amorim, Nguyen, Wigard, \& Mogensen, 2017). To overcome that risk, developments in GPS-spoofing security and geo-fencing have taken place (Sathyamoorthy, 2015). In an attempt to automate the flight control and make operations safer, researchers have advanced in developing autopilot and self-collision avoidance features into UAVs. Despite the progress in implementing safety measures to reduce safety hazards pertaining to UAV usage, the safe-to-crash paradigm is assumed to be far-fetched from accomplishing the required safety standards (Afman et al., 2018). A research study directed towards exploring the undiscovered safety risks of UAVs indicated that the existing basic safety features do not necessarily fulfil the construction industries' safety requirements and expectations (Namian et al., 2021a). The results of that study further revealed that necessary control measures are not employed on job sites and a substantial portion of the workforce does not have first-hand familiarity with the UAVs.

\section{Conclusions}

The advancement in technology is rapidly shifting the shape of integrated project delivery and the emergence of UAVs has made a handful of construction activities easier and cheaper, if not all. As a result, the rate of UAV integration into construction job site is increasing to survive in this modern environment saturated with cutting-edge technologies. As the construction industry evolves sustaining a wide range of occupational and operational complexities, the rapid integration of UAVs adds more uncertainty to it. The presented information of this paper suggests that adopting UAVs can expose the workforce to a wide variety of occupational safety risks and a significant portion of the workforce is unfamiliar with the practical use of UAVs. Therefore, the safety knowledge of UAV-based construction applications and related risks is insufficient. Unless systematic understanding and successful hazard prevention techniques are implemented, a high risk of exposure to the workforce remains unaccounted for. This review paper enables construction researchers, managers, engineers, superintendents, and safety officers to identify and address the risks affiliated with UAVs operated in the job site environments, which will aid the construction industry to better understand and effectively manage the UAV-related safety risks. UAVs must be taken into consideration for sufficient usage and experiments before actually incorporating them into the job sites for better and effective preparedness. Researchers were limited to the amount of synthesized literature reviewed for this study, but there is still more to investigate and understand about human-machine interrelations and how they shift in various domains of construction. Further investigations should also focus on developing construction-specific UAV training programs and guidelines for pilots and workers. 


\section{References}

Abuswer, M., Amyotte, P., Khan, F., \& Morrison, L. (2013). An optimal level of dust explosion risk management: Framework and application. Journal of Loss Prevention in the Process Industries, 26(6), 1530-1541. https://doi.org/10.1016/j.jlp.2013.08.018

Afman, J.-P., Ciarletta, L., Feron, E., Franklin, J., Gurriet, T., \& Johnson, E. N. (2018). Towards a New Paradigm of UAV Safety. ArXiv:1803.09026 [Cs]. Retrieved from http://arxiv.org/abs/1803.09026

Asadi, K., Kalkunte Suresh, A., Ender, A., Gotad, S., Maniyar, S., Anand, S., ... Wu, T. (2020). An integrated UGV-UAV system for construction site data collection. Automation in Construction, 112, 103068. https://doi.org/10.1016/j.autcon.2019.103068

Belcastro, C. M., Foster, J., Newman, R. L., Groff, L., Crider, D. A., \& Klyde, D. H. (2014). Preliminary Analysis of Aircraft Loss of Control Accidents: Worst Case Precursor Combinations and Temporal Sequencing. In AIAA SciTech Forum. AIAA Guidance, Navigation, and Control Conference (Vols. 10). American Institute of Aeronautics and Astronautics. https://doi.org/10.2514/6.2014-0612

Belcastro, C., Newman, R., Evans, J., Klyde, D., Barr, L., \& Ancel, E. (2017). Hazards Identification and Analysis for Unmanned Aircraft System Operations. https://doi.org/10.2514/6.2017-3269

Benjamin, G. (2020). Drone culture: Perspectives on autonomy and anonymity. AI \& SOCIETY. https://doi.org/10.1007/s00146-020-01042-7

Charlesraj, V. P. C., \& Rakshith, N. (2020). Stakeholder Perspectives on the Adoption of Drones in Construction Projects. ISARC Proceedings, 1227-1234. Kitakyushu, Japan: IAARC.

Clarke, R. (2014). Understanding the drone epidemic. Computer Law \& Security Review, 30(3), 230246. https://doi.org/10.1016/j.clsr.2014.03.002

Coker, C. (2013). Technology is making man the weakest link in warfare. The Financial Times.

Craik, F. I. M. (2014). Effects of distraction on memory and cognition: A commentary. Frontiers in Psychology, 5, 841. https://doi.org/10.3389/fpsyg.2014.00841

Federal Aviation Administration, (2020). FAA Aerospace Forecast Fiscal Years 2020-2040. Available from: https://www.faa.gov/data_research/aviation/aerospace_forecasts/media/FY202040_FAA_Aerospace_Forecast.pdf

Federal Aviation Administration, (2019). Press Release - U.S. Department of Transportation Issues Proposed Rule on Remote ID for Drones. Retrieved from https://www.faa.gov/news/press_releases/news_story.cfm?newsId=24534

Gheisari, M., \& Esmaeili, B. (2019). Applications and requirements of unmanned aerial systems (UASs) for construction safety. Safety Science, 118, 230-240. https://doi.org/10.1016/j.ssci.2019.05.015 
Guo, B. H. W., Yiu, T. W., \& González, V. A. (2015). Identifying behaviour patterns of construction safety using system archetypes. Accident Analysis \& Prevention, 80, 125-141. https://doi.org/10.1016/j.aap.2015.04.008

Hinze, J., Thurman, S., \& Wehle, A. (2013). Leading indicators of construction safety performance. Safety Science, 51(1), 23-28. https://doi.org/10.1016/j.ssci.2012.05.016

Kas, K. A., \& Johnson, G. K. (2020). Using unmanned aerial vehicles and robotics in hazardous locations safely. Process Safety Progress, 39(1). https://doi.org/10.1002/prs.12066

Kovacs, I., Amorim, R., Nguyen, H. C., Wigard, J., \& Mogensen, P. (2017). Interference Analysis for UAV Connectivity over LTE Using Aerial Radio Measurements. 2017 IEEE 86th Vehicular Technology Conference (VTC-Fall), 1-6. Toronto, ON, Canada.

Martinez, J. G., Gheisari, M., \& Alarcón, L. F. (2020). UAV Integration in Current Construction Safety Planning and Monitoring Processes: Case Study of a High-Rise Building Construction Project in Chile. Journal of Management in Engineering, 36(3), 05020005.

McCabe, B., Hamledari, H., Shahi, A., Zangeneh, P., \& Azar, E. R. (2017). Roles, benefits, and challenges of using UAVs for indoor smart construction applications. In Computing in Civil Engineering 2017 (pp. 349-357). https://ascelibrary.org/doi/abs/10.1061/9780784480830.043

Menouar, H., Guvenc, I., Akkaya, K., Uluagac, A. S., Kadri, A., \& Tuncer, A. (2017). UAV-Enabled Intelligent Transportation Systems for the Smart City: Applications and Challenges. IEEE Communications Magazine, 55(3), 22-28. https://doi.org/10.1109/MCOM.2017.1600238CM

Namian, M., Albert, A., \& Feng, J. (2018). Effect of distraction on hazard recognition and safety risk perception. Journal of Construction Engineering and Management, 144(4), 04018008. DOI: 10.1061/(ASCE)CO.1943-7862.0001459

Namian, M., Khalid, M., Wang, G. C., \& Turkan, Y. (2021a). Revealing Safety Risks of Unmanned Aerial Vehicles in Construction. Presented at the 100th Annual Meeting Transportation Research Board. Retrieved from https://trid.trb.org/view/1759453

Namian, M., Khalid, M., Wang, G., \& Turkan, Y. (2021b). Revealing Safety Risks of Unmanned Aerial Vehicles in Construction. Transportation Research Record. [In press]

Namian, M., Zuluaga, C. M., \& Albert, A. (2016). Critical factors that impact construction workers' hazard recognition performance. Construction Research Congress 2016, 2762-2772. San Juan, Puerto Rico: American Society of Civil Engineers.

Neff P, Garman KE (2016). Identifying and Mitigating Human Factors Errors in Unmanned Aircraft Systems. In: 16th AIAA Aviation Technology, Integration, and Operations Conference. Albuquerque, NM , USA. American Institute of Aeronautics and Astronautics.

Occupational Safety and Health Administration, (2018). OSHA's use of Unmanned Aircraft Systems in Inspections. [cited $2020 \mathrm{Jan}$ 4]. Available from: https://www.osha.gov/memos/2018-05-18/oshas-useunmanned-aircraft-systems inspections

Opfer, N. D., \& Shields, D. R. (2014). Unmanned aerial vehicle applications and issues for construction. In 121st ASEE Annual Conference and Exposition, 1-16. Indianapolis, IN, USA. 
Parush, A. (2006). Human Errors in UAV Takeoff and Landing: Theoretical Account and Practical Implications. In N. J. Cooke, H. L. Pringle, H. K. Pedersen, \& O. Connor (Eds.), Human Factors of Remotely Operated Vehicles (pp. 91-103). Emerald Group Publishing Limited. https://doi.org/10.1016/S1479-3601(05)07007-4

Rao, B., Gopi, A. G., \& Maione, R. (2016). The societal impact of commercial drones. Technology in Society, 45, 83-90. https://doi.org/10.1016/j.techsoc.2016.02.009

Sathyamoorthy, D. (2015). A review of security threats of unmanned aerial vehicles and mitigation steps. The Journal of Defence and Security, 6.

Schenkelberg, F. (2016). How reliable does a delivery drone have to be? 2016 Annual Reliability and Maintainability Symposium (RAMS), 1-5. Tucson, AZ, USA.

Trinh, M. T., \& Feng, Y. (2020). Impact of project complexity on construction safety performance: Moderating role of resilient safety culture. Journal of Construction Engineering and Management, 146(2), 04019103. https://doi.org/10.1061/(ASCE)CO.1943-7862.0001758

U.S. Bureau of Labor Statistics. (2020). Number and rate of fatal work injuries, by industry sector. Retrieved February 17, 2021, from https://www.bls.gov/charts/census-of-fatal-occupationalinjuries/number-and-rate-of-fatal-work-injuries-by-industry.htm

Wang, G., Hollar, D., Sayger, S., Zhu, Z., Buckeridge, J. S., Li, J., ... Hu, W. (2016). Risk Considerations in the Use of Unmanned Aerial Vehicles in the Construction Industry. Journal of Risk Analysis and Crisis Response, (6:4), p.165-177. Retrieved from https://thescholarship.ecu.edu/handle/10342/6067

York, D. D., Al-Bayati, A. J., \& Al-Shabbani, Z. Y. (2020). Potential Applications of UAV within the Construction Industry and the Challenges Limiting Implementation. 31-39. https://doi.org/10.1061/9780784482889.004

Zhang, M., Murphy, L. A., Fang, D., \& Caban-Martinez, A. J. (2015). Influence of fatigue on construction workers' physical and cognitive function. Occupational Medicine, 65(3), 245-250. https://doi.org/10.1093/occmed/kqu215 